\title{
Production of an aberrant splice variant of CCL5 is not caused by genetic mutation in the mammary glands of mastitis-infected Holstein cows
}

\author{
LING YANG $^{1^{*}}$, RUIQING GUO ${ }^{1,2^{*}}$, ZHIHUA JU ${ }^{2}$, XIUGE WANG ${ }^{2}$, QIANG JIANG ${ }^{2}$, YONG LIU $^{1,2}$, \\ $\mathrm{HAN}_{\mathrm{ZHAO}}{ }^{2,3}$, KAILI HE ${ }^{1}$, JIANBIN $\mathrm{LI}^{2}$ and JINMING HUANG ${ }^{2,3}$ \\ ${ }^{1}$ College of Life Sciences and Food Engineering, Hebei University of Engineering, Handan, Hebei 056021; \\ ${ }^{2}$ Dairy Cattle Research Center, Shandong Academy of Agricultural Sciences, Jinan, Shandong 250100; \\ ${ }^{3}$ College of Life Sciences, Shandong Normal University, Jinan, Shandong 250014, P.R. China
}

Received July 26, 2018; Accepted February 21, 2019

DOI: $10.3892 / \mathrm{mmr} .2019 .10103$

\begin{abstract}
Genetic mutations, including single nucleotide polymorphisms (SNPs), result in aberrant alternatively splicing of gene and involves in susceptibility of inflammatory diseases, including bovine mastitis. C-C motif chemokine ligand 5 (CCL5) is an immune-associated gene, but its alternative splicing (AS) mechanism of gene expression has not yet been understood. The present study identified the splice variant of CCL5 and the compared differential expression of various transcripts between healthy and mastitic mammary gland tissue from cows. A novel transcript lacking exon 2 with a deletion of 112 bp (referred to as CCL5-AS) was identified in the mammary gland. The expression of CCL5-AS was lower compared with CCL5-reference in the healthy and mastitic mammary tissues. A total of two novel SNPs ( . 1647 C>T and g.1804 G>A) were identified in exon 2 of CCL5. Using the splicing mini-gene reporter assay in bovine mammary epithelial cells (MAC-T) and 293T cells, it was confirmed that the production of CCL5-AS was not caused by the two SNPs. The present findings suggested that alternative splicing is one of the mechanisms of CCL5 expression regulation and is involved in mastitis infection, but that genetic mutation was not responsible for the generation of the abnormal transcript of CCL5 in cows.
\end{abstract}

\section{Introduction}

Mastitis is the most common infectious disease in dairy cows, and it remains a major challenge to the global dairy

Correspondence to: Dr Jinming Huang, Dairy Cattle Research Center, Shandong Academy of Agricultural Sciences, 159 Industry North Road, Jinan, Shandong 250100, P.R. China

E-mail: huangjinm@sina.com

*Contributed equally

Key words: alternative splicing, bovine mastitis, C-C motif chemokine ligand 5, single nucleotide polymorphisms industry (1). Mastitis is an inflammation of the parenchyma of the mammary gland, and is caused by different pathogens, including Staphylococcus aureus, Escherichia coli and Streptococcus uberis $(2,3)$. Challenging or natural infection by different pathogens results in an alteration in the mRNA expression of a subset of immune-associated genes in cows (4-6). Immune responses to infecting pathogens in the bovine mammary gland are very complex. Therefore, a coordinated response exists between the resident, recruited and inducible immune factors, including chemokines (5). Upon bacterial stimulation, bovine mammary epithelial cells are activated and are able to release neutrophil-mobilizing chemokines, contributing to different immune and inflammatory responses of the mammary gland (7). C-C motif chemokine ligand 5 (CCL5), also termed RANTES, is upregulated in bovine mammary epithelial cells following stimulation by $E$. coli and crude lipopolysaccharide, but not by $S$. aureus culture supernatant (8). By contrast, in a previous study, it was identified that the CCL5 gene was downregulated in S. aureus mastitis-infected mammary glands by 1.61 -fold compared with non-infected tissue in cows (6). CCL5 belongs to the C-C chemokine family (9). Functional CCL5 serves an active role in recruiting a variety of leukocytes, including T-cells, macrophages, eosinophils and basophils to inflammatory sites (10).

Alternative splicing (AS) of a gene may generate numerous mature mRNA isoforms in higher eukaryotes (11). A limited number of genes are able to generate vast numbers of proteins via AS of eukaryotic transcripts in cells, which has notable physiological functions in the different developmental processes in humans (12). According to the Alternative Splicing Graph Server (ASGS), in a genome-wide analysis only $21 \%(4,567$ of 21,755$)$ bovine genes were identified to be alternatively spliced (13). Using Illumina paired-end RNA sequencing and bioinformatics methods, a previous study identified that 8,549 (52.62\%) and 8,325 (51.24\%) unigenes were alternatively spliced, exhibiting 34,523 and 30,410 AS events in terms of exon skipping, intron retention, alternative 5 ' splicing and alternative 3 ' splicing patterns in healthy and mastitic tissues, respectively (6). Among them, CCL5 
(ENSBTAG00000007191) exhibited an exon-skipping splicing pattern, which was specific to the mastitis-infected individual (6).

Single nucleotide polymorphisms (SNPs) are the most frequent type of variation, and major contributors to natural splice variants (14). Mutations or variations in either cis-acting elements or trans-acting factors result in aberrant splicing and abnormal protein production (15), which are associated with certain important diseases, including bovine mastitis $(3,16,17)$. However, alternative splicing and expression of the bovine CCL5 gene has not been elucidated, to the best of our knowledge. In the present study, splice variants, transcript expression, and splicing-associated SNPs of the CCL5 gene were investigated in healthy and mastitic mammary glands in Holstein cows.

\section{Materials and methods}

Animals and experimental design. All experiments were conducted according to the National Standards for Laboratory Animals Guideline for Ethical Review of Animal Welfare of China (GB/T 35892-2018), and were approved by the Animal Care and Use Committee of the Dairy Cattle Research Center, Shandong Academy of Agricultural Sciences (Jinan, China). Mammary tissue samples were collected from eight healthy and eight mastitic Chinese Holstein cows (650-750 kg) at 2.8 to 3.0 years of age during their first lactation at a commercial slaughter plant in Jinan, China. The duration of the experiment was 3 months, and no animal succumbed during that time. Prior to the collection of mammary tissues, the animal health and behavior were monitored daily. The mastitic cows were selected according to their clinical symptoms, and the mastitis infections were caused only by $S$. aureus. The healthy cows were not affected by pathogen infection and had no clinical symptoms (heat, pain, redness, swelling of the udder and milk clotting). The mammary tissues were sampled at the time of slaughter and frozen immediately in liquid nitrogen.

Identification of the splice variants of the bovine CCL5 gene. An E.Z.N.A ${ }^{\circledR}$ Mollusc RNA kit (Omega Bio-Tek, Inc., Norcross, GA, USA) was used to extract the total RNA from the mammary samples, and cDNA was synthesized using a PrimeScript $^{\mathrm{TM}}$ Reverse Transcription (RT) reagent kit with gDNA Eraser (Perfect Real Time; Takara Biotechnology Co., Ltd., Dalian, China), according to the manufacturer's protocol. The primer sequences of the CCL5 gene (GenBank accession no. NM_175827.2) and KB-SNP1 were designed using Primer Premier 5.0 (Premier Biosoft International, Palo Alto, CA, USA) and Primer-BLAST software (www.ncbi. nlm.nih.gov/tools/primer-blast/; Table I), and synthesized by Beijing TsingKe Biological Technology Co., Ltd. (Beijing, China). The polymerase chain reaction (PCR) was performed as follows: $94^{\circ} \mathrm{C}$ for $4 \mathrm{~min}, 94^{\circ} \mathrm{C}$ for $30 \mathrm{sec}, 68^{\circ} \mathrm{C}$ for $30 \mathrm{sec}$, $72^{\circ} \mathrm{C}$ for $35 \mathrm{sec}(35 \mathrm{cycles})$, and $72^{\circ} \mathrm{C}$ for $10 \mathrm{~min}$. DNA bands were separated by $1 \%$ agarose gel electrophoresis and eluted using a Universal DNA Purification kit (Tiangen Biotech Co., Ltd., Beijing, China). The method of identifying the splice variants was performed as described previously (18). The PCR products were subcloned into the pEASY-T3 cloning vector
(TransGen Biotech Co., Ltd., Beijing, China) following purification, and the mixture was transformed into E. coli $\mathrm{DH} 5 \alpha$ competent cells (Takara Biotechnology Co., Ltd.). The positive clones were selected and sequenced by BGI LifeTech Co., Ltd. (Beijing, China) and DNAMAN v5.2.2 software (Lynnon LLC, San Ramon, CA, USA) was used to identify the possible splice variants through multiple sequence alignments with the CCL5-reference mRNA.

Quantification of abundance of CCL5 transcript variants. An E.Z.N.A ${ }^{\circledR}$ Mollusc RNA kit was used to extract the total RNA from the mammary samples, and cDNA was synthesized as aforementioned. SYBR-Green PCR Master Mix (Tiangen Biotech Co., Ltd.) was used for RT-quantitative (q)PCR in a BioRad CFX96 Real-Time System (Bio-Rad Laboratories, Inc., Hercules, CA, USA), according to the manufacturer's protocol. The primer sequences for CCL5 and $\beta$-actin (GenBank accession no. NM_173979.3; www.ncbi.nlm.nih.gov/genbank) were designed using Primer Premier 5.0 and Primer-BLAST software, and the primer sequences of CCL5-reference and CCL5-AS (Table II) were designed based on the results for the splice variants of the CCL5 gene. The primer sequences were synthesized by Beijing TsingKe Biological Technology Co., Ltd. The relative expression values for the RT-qPCR assay were calculated with $\beta$-actin as the endogenous control, and the template that did not undergo the reverse transcription reaction served as the negative control. qPCR was performed as follows: $95^{\circ} \mathrm{C}$ for $30 \mathrm{sec}, 95^{\circ} \mathrm{C}$ for $15 \mathrm{sec}, 60^{\circ} \mathrm{C}$ for $30 \mathrm{sec}$, $72^{\circ} \mathrm{C}$ for $35 \mathrm{sec}$ ( 40 cycles).

Prediction of CCL5 splice variant. Exonic splice enhancer(ESE) finder 3.0 (http://rulai.cshl.edu/cgi-bin/tools/ESE3/esefinder. cgi) was used to predict the alterations in the binding site of the splicing factor, and the alternative splice site prediction was achieved with ASSP (http://wangcomputing. com/assp/index.html).

CCL5 splicing mini-gene reporter assay. The 598 bp genomic fragment of the CCL5 gene from bovine genomic DNA, including intron 1, intron 2 and exon 2, was amplified to evaluate the in vitro splicing with mini-genes, and the primer sequences of KB-SNP1 are presented in Table I. The segment with the wild-type, AA, or the mutant type, TA, of the CCL5 gene was cloned into the pSPL3 vector (Invitrogen; Thermo Fisher Scientific, Inc., Waltham, MA, USA) following digestion with EcoRI and XhoI. The clones were transformed into Trans5a cells (TransGen Biotech Co., Ltd.) which were plated on agar containing $100 \mathrm{mg} / \mathrm{ml}$ ampicillin, and incubated at $37^{\circ} \mathrm{C}$ overnight. The positive colonies were cultured in lysogeny broth at $37^{\circ} \mathrm{C}$ overnight, and an Endo-free Plasmid Mini kit II (Omega Bio-Tek, Inc.) was used to isolate the plasmids according to the manufacturer's protocols. The presence of the correct sequences was verified through sequencing constructs by Beijing TsingKe Biological Technology Co., Ltd.

Bovine mammary epithelial cells (MAC-T) and 293T cells were cultured in Dulbecco's modified Eagle's medium (Gibco; Thermo Fisher Scientific, Inc.) containing penicillin/streptomycin $(100 \mathrm{IU} / \mathrm{ml} ; 0.1 \mathrm{mg} / \mathrm{ml})$ with $10 \%$ fetal bovine serum in an atmosphere of $5 \% \mathrm{CO}_{2}$ in air at $37^{\circ} \mathrm{C}$. The cells were 
Table I. Primers used for screening SNPs.

\begin{tabular}{llcc}
\hline Primers & \multicolumn{1}{c}{ Primer sequences } & Product size, bp & Annealing temperature, ${ }^{\circ} \mathrm{C}$ \\
\hline CCL5 mRNA & F: CTGCGCTCCTGCTTCTG & 566 & 58 \\
KB-SNP1 & R: CCAGTGAGGGACCGAGAT & 598 & 58 \\
& F GGCGGAGGAACTAAGACCAG & & \\
\hline
\end{tabular}

F, forward; R, reverse; SNP, single nucleotide polymorphism; CCL5, C-C motif chemokine ligand 5.

Table II. Primers used in the reverse transcription-quantitative polymerase chain reaction.

\begin{tabular}{llcc}
\hline Primers & \multicolumn{1}{c}{ Primer sequences } & Product size, bp & Annealing temperature, ${ }^{\circ} \mathrm{C}$ \\
\hline$\beta$-actin & F: CACAATGAAGATCAAGATCATC & 173 & 60 \\
& R: CTAACAGTCCGCCTAGAAGCA & & 60 \\
CCL5-reference & F: CACCCACGTCCAGGAGTATTT & 174 \\
R: GCAAGTTCAGGTTCAAAACG & F: GCTTCTGCCTCCCATCTTTAT & 142 \\
& R: AAAGTTGGCGCAAGTTCAGG & & 60 \\
\hline
\end{tabular}

CCL5, C-C motif chemokine ligand 5; AS, alternative splicing; F, forward; R, reverse.

transferred to six-well culture plates, and transfected with $4 \mu \mathrm{g}$ of wild-type or mutant mini-gene constructs using Lipofectamine ${ }^{\circledR} 2000$ Transfection Reagent (Invitrogen; Thermo Fisher Scientific, Inc.) for $5 \mathrm{~h}$ in Opti-MEM medium (Gibco; Thermo Fisher Scientific, Inc.), according to manufacturer's protocol, when the cells had grown to $80-85 \%$ confluence. A subset of cells were transfected with pSPL3 lacking the CCL5 insert, and other cells were treated with only transfection reagent as the control.

The cells were collected at $24 \mathrm{~h}$ post-transfection, and the total RNA was extracted, reverse transcribed into cDNA, and PCR was performed as described above. The pSPL3 vector specific primers were SD6 (5'-TCTGAGTCACCTGGACAA CC-3') and SA2 (5'-ATCTCAGTGGTATTTGTGAGC-3'). The PCR products were separated on $2 \%$ agarose gels, and the DNA bands were extracted with a Gel Extraction kit (Omega Bio-Tek, Inc.) and analyzed by direct sequencing by Beijing TsingKe Biological Technology Co., Ltd.

Statistical analyses. Relative quantification of CCL5-reference and CCL5-AS mRNA expression was performed using the $2^{-\Delta \Delta \mathrm{Cq}}$ analysis method for relative real-time PCR (19). The data for the relative expression levels of CCL5-reference and CCL5-AS mRNA were analyzed using a completely randomized design with eight animals per group, via a mixed-model analysis of variance using Proc Mixed in SAS (version 9.1; SAS Institute, Cary, NC, USA). The comparisons among the relative expression levels of the different groups were performed using the Duncan method, controlling the experiment wise type \pm error equal to 0.05 . Data are presented as least squares means. $\mathrm{P}<0.05$ was considered to indicate a statistically significant difference.

\section{Results}

Bovine CCL5 splice variants identified in mammary glands. The cDNA from mammary tissues was used to amplify the full-length CCL5 transcript with specific primers for CCL5 cDNA (Table I). As a result, two PCR amplification fragments were detected in the mastitis mammary gland tissues, while only one fragment was detected in the healthy tissues (Fig. 1A). A total of two PCR bands of different sizes were recovered and subsequently sequenced. The common PCR fragment was the expected CCL5 product (566 bp), and the shorter PCR fragment was a novel splice variant with a size of $454 \mathrm{bp}$. By comparing with the reference genomic sequence of the bovine CCL5 gene (GenBank accession no. NC_007317.6) and the reference mRNA sequence (GenBank accession no. NM_175827.2), it was observed that the shorter splice variant, designated CCL5 transcript variant CCL5-AS, lacked the entirety of exon 2 with a 112 bp deletion (Fig. 1B and C). Furthermore, the putative protein of CCL5-AS was missing the key Chemokine_CC domain when compared with the CCL5-reference protein (Fig. 1D), indicating the loss of the normal function of CCL5 in the immune and inflammatory processes.

Transcription pattern of the bovine CCL5 splice variants in healthy and mastitic mammary gland tissues. Through the RT-qPCR, the relative expression levels of CCL5-reference and CCL5-AS were compared in the mammary gland tissues of cows. The expression of CCL5-reference mRNA in the healthy tissue was significantly higher compared with that in the mastitic tissue. Moreover, it was demonstrated that the CCL5-reference was the most abundant transcript in the 

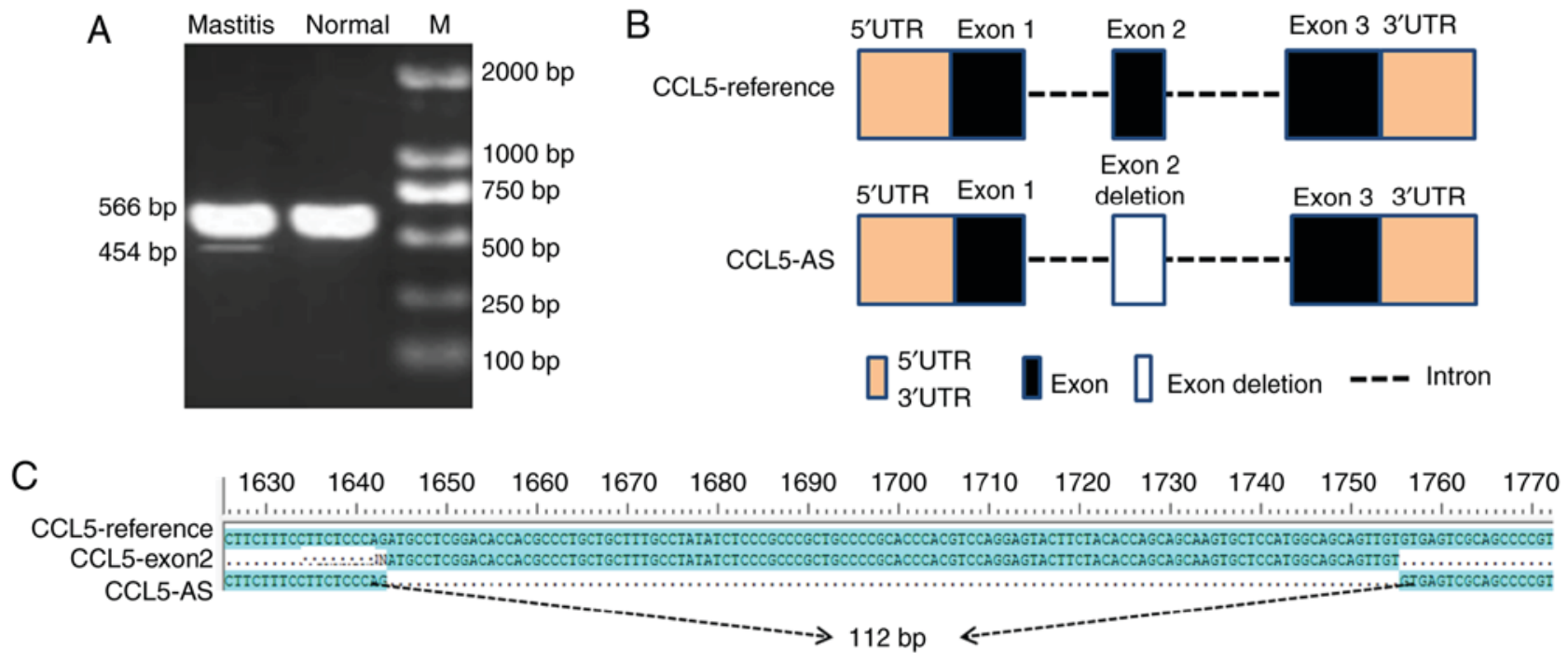

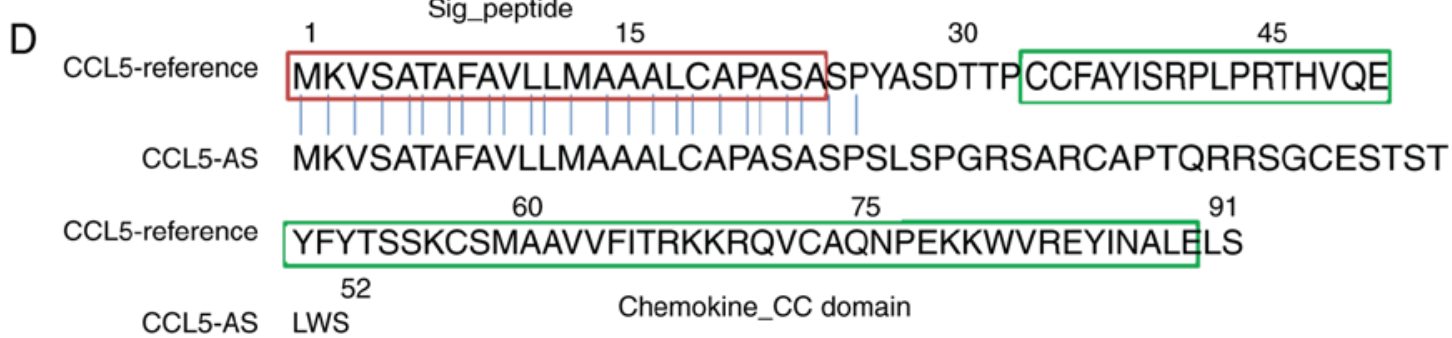

Figure 1. Alternative splicing pattern of the bovine CCL5 in the mammary glands. (A) Polymerase chain reaction products of the CCL5 gene expressed in bovine mammary tissues. The $566 \mathrm{bp}$ product corresponded to the transcript of CCL5-reference; the $454 \mathrm{bp}$ band corresponded to the transcript of CCL5-AS (B) Comparison of the genomic structures of bovine CCL5-reference and CCL5-AS using the DNAMAN software. (C) The CCL5-AS splice variant lacked exon 2 (112 bp). (D) Domains of CCL5-reference and putative CCL5-AS proteins. UTR, untranslated region; AS, alternative splicing; M, marker; CCL5, C-C motif chemokine ligand 5.

healthy and mastitic tissues, which is consistent with the RT-PCR results. The novel transcript CCL5-AS was expressed in the mastitic mammary tissues with a low abundance, whereas it was undetectable in the normal tissues (Fig. 2). These results supported the hypothesis that CCL5-AS was specific to mastitic tissue.

Putative splicing-related SNPs identified in exon 2 and intron 2 of CCL5. To analyze the molecular basis of the aberrant CCL5-AS splice variant, a pair of primers (KB-SNP1; Table I) was designed to amplify a $598 \mathrm{bp}$ fragment harboring exon 2 of CCL5. A total of two novel SNPs, one SNP (g.1647C $>$ T) in exon 2 (a synonymous mutation) and the other SNP (g.1804G $>$ A) in the intron 2 region, were identified in the Holstein cow population by sequencing the PCR amplification products (Fig. 3A). To investigate whether the SNPs affected the alternative splicing of exon 2, ESEfinder 3.0 software was used to predict alterations in the splice sites and factors in the ESE motif. The prediction demonstrated that the introduction of allele $\mathrm{T}$, relative to allele $\mathrm{C}$, in the locus g. $1647 \mathrm{C}>\mathrm{T}$ resulted in the deletion of two binding sites of auxiliary splicing proteins, serine/arginine-rich splicing factor 1 (SRSF1) and SRSF1 (IgM-BRCA1); however, the introduction of allele $A$, relative to allele $\mathrm{G}$, in the locus g.1804G $>$ A led to the deletion of two IgM-BRCA1 binding sites, and increased the SRSF5 (SRp40) binding site (Fig. 3B). A total of two SNPs (g.1647C $>$ T and g.1804G $>$ A) located in

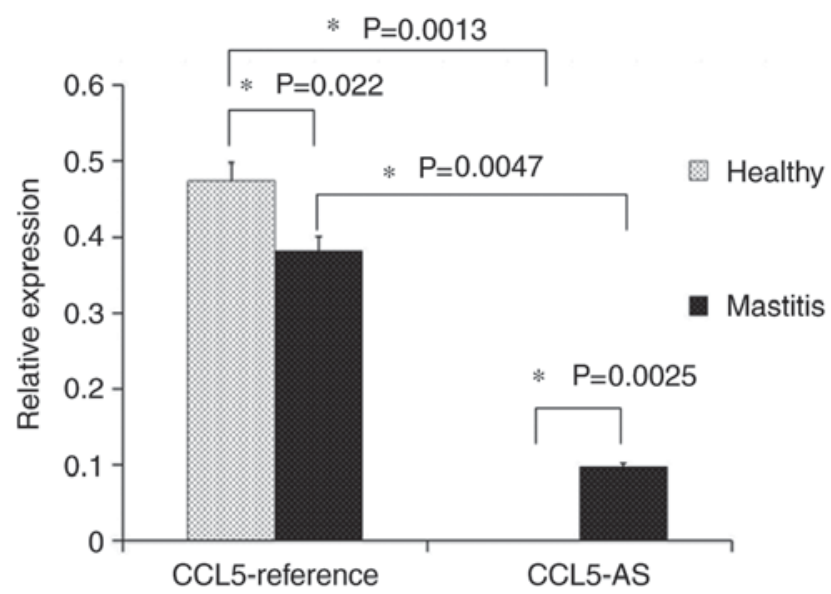

Figure 2. Relative expression levels of CCL5-reference and CCL5-AS mRNA in the mammary gland tissues from healthy and mastitis-infected cows. The $\mathrm{x}$-axis of the graph indicates the relative expression levels of CCL5-reference and CCL5-AS mRNA, and the y-axis of the graph indicates CCL5-reference and CCL5-AS mRNA. "P<0.05. AS, alternative splicing; CCL5, C-C motif chemokine ligand 5.

the ESE motif region were in complete linkage disequilibrium, constituting two haplotypes (C-G and T-A) of SNPs (g.1647C $>\mathrm{T}$ and g.1804G $>\mathrm{A}$ ), which may putatively induce the production of a novel splice variant of the bovine CCL5 gene. 
A

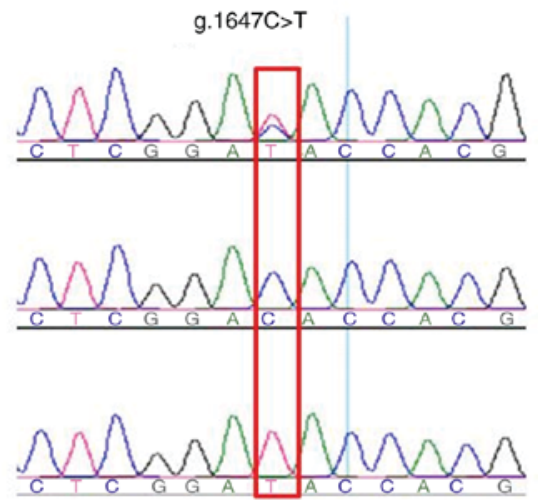

g. $1804 G>A$

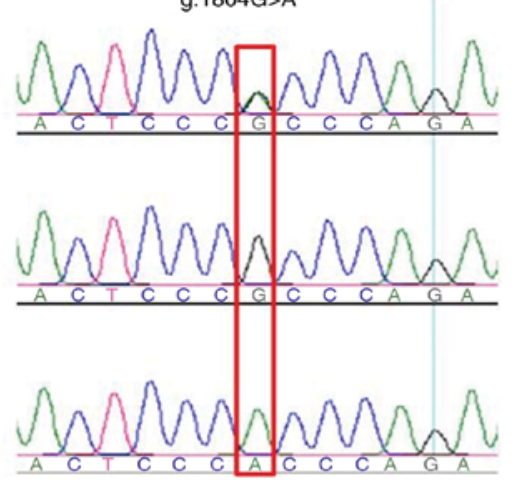

g. $1647 \mathrm{C}>\mathrm{T}-\mathrm{T}$

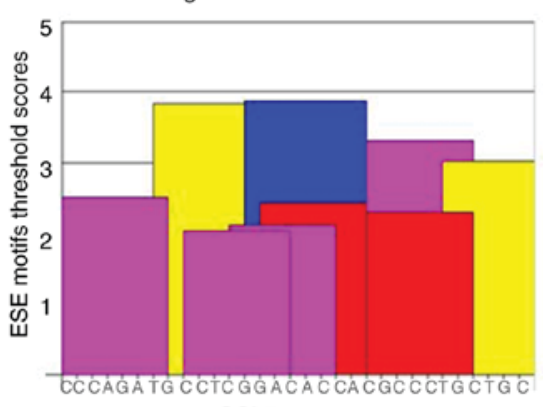

CCL5 sequence
B

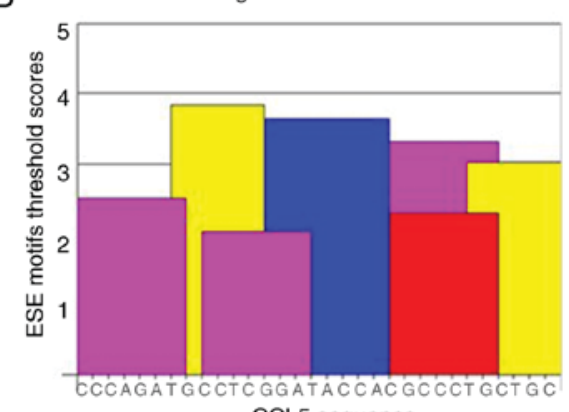

CCL5 sequence

\begin{tabular}{|c|c|c|c|c|c|}
\hline SNP SR prote & $\begin{array}{c}\text { SRSF1 } \\
\text { Position/site/score }\end{array}$ & $\begin{array}{l}\text { SRSF1 (IgM-BRCA1) } \\
\text { Position/site/score }\end{array}$ & $\begin{array}{c}\text { SRSF2 } \\
\text { Position/site/score }\end{array}$ & $\begin{array}{c}\text { SRSF5 } \\
\text { Position/site/score }\end{array}$ & $\begin{array}{c}\text { SRSF6 } \\
\text { Position/site/score }\end{array}$ \\
\hline \multirow{4}{*}{ g. $1647 \mathrm{C}>\mathrm{T}-\mathrm{C}$} & $14(-18) / G A C A C C A / 2.43404$ & $1(-31) / \mathrm{CCCAGAT} / 2.50560$ & 13(-19)/GGACACCA 13.87423 & & $7(-25) / T G C C T C / 3.83191$ \\
\hline & $21(-11) / C G C C C T G / 2.30151$ & $9(-23) / \mathrm{CCTCGGA} / 2.03828$ & & & $26(-6) /$ TGCTGC/3.01803 \\
\hline & & $12(-20) / C G G A C A C / 2.11382$ & & & \\
\hline & & $21(-11) / C G C C C T G / 3.31465$ & & & \\
\hline \multirow{3}{*}{ g. $1647 \mathrm{C}>\mathrm{T}-\mathrm{T}$} & $21(-11) / \mathrm{CGCCCTG} / 2.30151$ & 1(-31)/CCCAGAT/2.50560 & 13(-19)/GGATACC 3.64338 & & $7(-25) / T G C C T C / 3.83191$ \\
\hline & & 9(-23)/CCTCGGA/2.03828 & & & $26(-6) /$ TGCTGC/3.01803 \\
\hline & & $21(-11) / C G C C C T G / 3.31465$ & & & \\
\hline
\end{tabular}

C

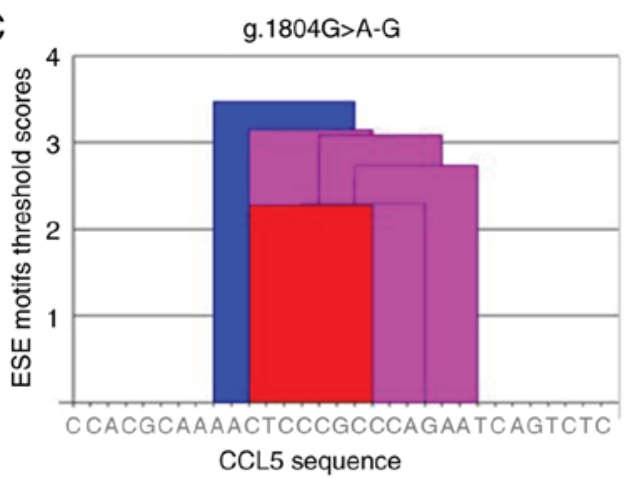

g.1804G>A-A

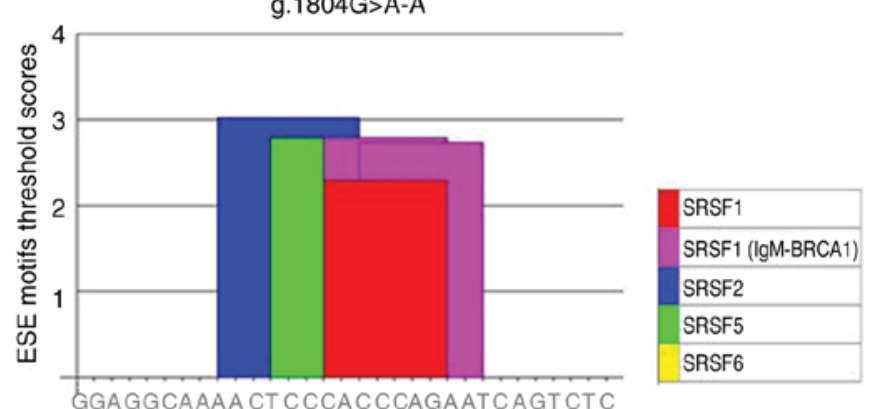

CCL5 sequence

\begin{tabular}{|c|c|c|c|c|}
\hline SNP $\quad \begin{array}{c}\text { SRSF1 protein } \\
\text { Position/site/score }\end{array}$ & $\begin{array}{l}\text { SRSF1(IgM-BRCA1) } \\
\text { Position/site/score }\end{array}$ & $\begin{array}{c}\text { SRSF2 } \\
\text { Position/site/score }\end{array}$ & $\begin{array}{c}\text { SRSF5 } \\
\text { Position/site/score }\end{array}$ & $\begin{array}{c}\text { SRSF6 } \\
\text { Position/site/score }\end{array}$ \\
\hline $11(-21) / \mathrm{CTCCCGC} / 2.6638$ & $11(-21) / \mathrm{CTCCCGC} / 3.13945$ & 9(-23)/AACTCCC /3.46345) & & \\
\hline g.1804G>A-G & 15(-17)/CGCCCA@3.07831 & & & \\
\hline & $17(-15) / C C C A G A A / 2.73329$ & & & \\
\hline
\end{tabular}

Figure 3. ESE motif threshold scores associated with CCL5 genotypes. (A) Sequencing identification of two SNPs (g.1647 C>T and g.1804 G>A). (B) SNP (g.1647 C $>$ T). The bar graphs represent scores above the threshold for the ESE motifs in the $\mathrm{C}$ or $\mathrm{T}$ allele of locus 1647. The red rectangles indicate the introduction of allele $\mathrm{T}$, relative to allele $\mathrm{C}$, in the locus g.1647C $>\mathrm{T}$, and thus the deletion of binding sites to the auxiliary splicing proteins SRSF1 and SRSF1 (gM-BRCA1). The blue ellipses indicate the change in a score of SRSF2 binding site. (C) SNP g.1804 G>A. The bar graphs represent scores above the threshold for the ESE motifs in the G or A allele of locus 1804. The red rectangles indicates the introduction of allele A, relative to allele G, in the locus g.1804G $>\mathrm{A}$, and thus the deletion of two IgM-BRCA1 binding sites, and an increase in the SRSF5 (SRp40) binding site. The blue ellipses indicate the changes in a position/site/score of SRSF1 binding site, a score of IgM-BRCA1 binding site and a score of SRSF2 binding site. ESE, exonic splice enhancer; CCL5, C-C motif chemokine ligand 5; SNP, single nucleotide polymorphism; SRSF, serine/arginine-rich splicing factor; BRCA1, BRCA1 DNA repair associated. 
A Amplification the segment with either wild-type or mutant CCL5 g. $1647 \mathrm{C}>\mathrm{T}$ and g. $1804 \mathrm{G}>\mathrm{A}$

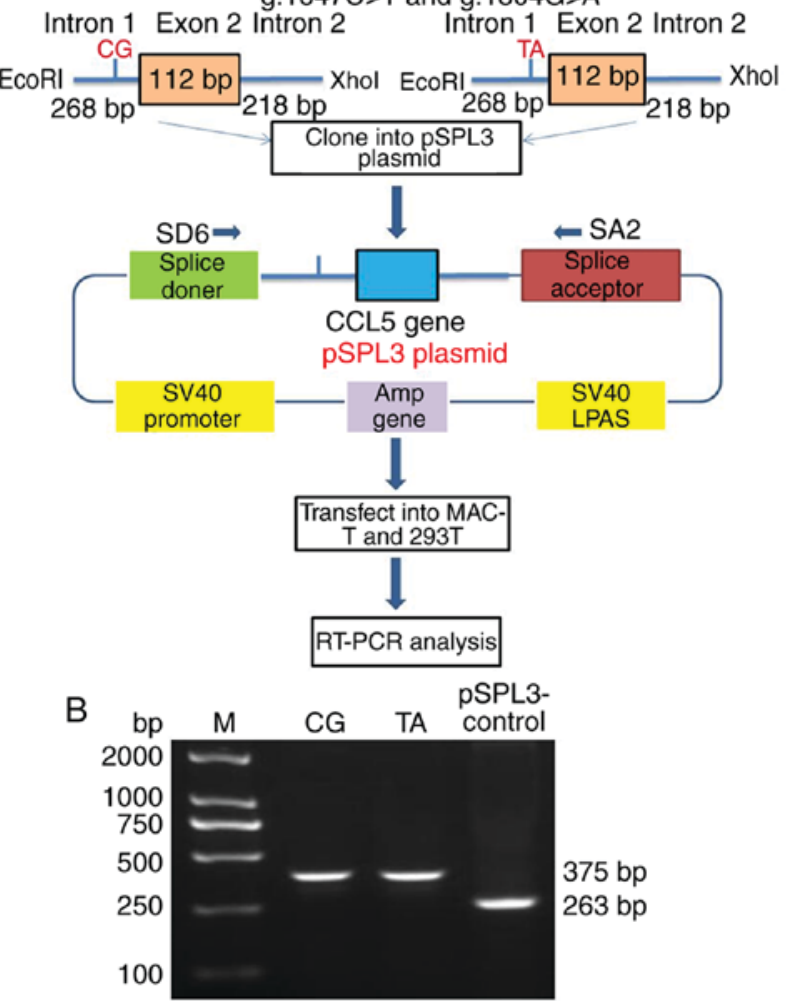

Figure 4. Protocol of splicing mini-gene reporter assay. (A) A total of two haplotypes (the wild haplotype C-G, g.1647 C>T-C and g.1804 G>A-G; and the mutant haplotype TA with two SNPs, g.1647 C>T-T and g.1804 G>A-A) generated the same splicing pattern of CCL5 in MAC-T and 293T cells. (B) RT-PCR analysis of the CCL5 spliced transcripts on a $2 \%$ agarose gel. The wild haplotype, CG, and the mutant type, TA, mini-gene reporters gave rise to the same $375 \mathrm{bp}$ fragment, while there was a $263 \mathrm{bp}$ fragment of pSPL3 vector in the MAC-T and 293T cells. Nonspecific amplification products were visible and sequenced. CCL5, C-C motif chemokine ligand 5; RT-PCR, reverse transcription-polymerase chain reaction; M, marker.

SNPs in the putative ESEs do not generate the novel splice transcript. To determine whether the two SNPs (g.1647C>T and g.1804G $>$ A) of CCL5 led to the abrogation of the predicted SR protein motifs and caused AS, the 598 bp genomic fragment spanning 268 bp of intron 1, 112 bp of exon 2 and 218 bp of intron 2 was amplified, which harbored the two haplotypes (C-G and T-A), and the two fragments were cloned into the pSPL3 vector (Fig. 4A). The CCL5 splicing mini-gene reporter assay demonstrated that transfecting the two plasmids, with the wild haplotype (C-G) and mutant haplotype (TA), respectively, into the $293 \mathrm{~T}$ cells produced the same RT-PCR amplification product with a size of $375 \mathrm{bp}$, while the negative pSPL3-control generated a product of $263 \mathrm{bp}$ (Fig. 4B). The results suggested that the haplotypes of the two SNPs (g.1647C>T and g.1804G >A) were not responsible for the production of CCL5-AS.

\section{Discussion}

The present study identified two splice variants and SNPs of CCL5, and confirmed its downregulation in the mammary glands from cows with clinical mastitis caused by natural infection with S.aureus. Furthermore, using the exon splicing mini-gene reporter assay, it was demonstrated that the candidate SNPs were not the cause of the generation of the aberrant transcript, CCL5-AS, in the mammary glands.

Mammary inflammation is a normal defensive response to bacterial invasion, and part of the subsequent direct immune response. When inflammation cannot be controlled in the mammary glands, mastitis occurs and leads to tissue damage. During the inflammatory response, processes including pathogen recognition, the release of pro-inflammatory cytokines and chemokines, rapid recruitment of immune cells from the vasculature to the infection site, and the subsequent removal of pathogens from the mammary glands, are of importance $(4,20)$. During the early stages of $E$. coli mastitis, the chemokine response is considered to be a decisive mechanism and serves a critical role in the inflammatory process (4). Following the challenge with $E$. coli, chemokine levels in milk whey and the density of polymorphonuclear neutrophils are markedly elevated in udder tissue (21). However, expression changes of the chemokine CCL5 gene in bovine mammary gland explants in response to peptidoglycan combined with lipotechoic acid, representing gram-positive bacteria (for example, S. aureus), have not been observed (20). In this study, the chemokine CCL5 was downregulated in the mammary glands in the late stages of natural mastitis infection. Differences in the CCL5 expression pattern may be due to different infection stages and model systems of cells, contributing to different immune responses to pathogens. CCL5 interacts with the G-protein-coupled receptors C-C motif chemokine receptor (CCR)1, CCR3 and CCR5 in various types of cells (22). Novel therapies for chronic inflammatory diseases target CCL5, which may decrease inflammatory responses and fibrosis (23). CCL5 is an immune-associated gene, which is expressed by T-lymphocytes, macrophages, platelets, synovial fibroblasts and the tubular epithelium (10). Therefore, CCL5 expression and its regulation in the mammary gland may be implicated in bovine mastitis infection and prevention.

AS may enhance transcriptomic and proteomic diversity in species ranging from bacteria to humans (24), and the regulation of AS is important for the determination of complex traits (25). In total, 92-94\% of human genes undergo AS, which leads to a single gene producing multiple mRNA and protein isoforms that have similar, distinct or even opposing functions (26). In the present study, a novel CCL5 transcript lacking exon 2 was expressed in the mammary gland tissue and was mastitis-specific, confirming a recent study and hypothesis (6). The results suggested that AS is one of the regulatory mechanisms underlying the repression of mRNA transcription and the loss of function of CCL5 at the late stage of mastitis infection, contributing to the severity of inflammation and tissue damage.

Different SNPs result in a variety of SRSF1 binding sites (27), and variations in the intronic SRSF1-binding site influences binding affinity and leads to the alteration of splicing, according to global cross-linking immunoprecipitation anlaysis (28). A number of studies have reported that SNPs in the exons or introns of immune-associated genes result in aberrant splicing and are associated with susceptibility to bovine mastitis $(3,16,17)$. In the present study, the in silico predicted results indicated that two SNPs may result in the deletion of two binding sites for SRSF1 and SRSF1 
(IgM-BRCA1), and increase the SRSF5 (SRp40) binding site. SRSF proteins are a battery of splicing regulatory proteins, which are implicated in numerous cellular functions under pathological and physiological conditions (29). Copy number variations (CNVs) may result in sporadic chromosomal microdeletion syndromes and Mendelian diseases (30). Insertions/deletions (indels) exert a strong influence on gene function, thus indels may be the 'driver mutations' in oncogenesis (31). CNVs and microRNA (miRNA) variants are associated with inherited retinal diseases in humans $(32,33)$. In the present study, a pSPL3 exon trapping system was used to verify the two SNPs, but the SNPs were not able to induce the production of CCL5-AS, which may be caused by indels, miRNA variants or CNVs. The molecular basis of the production of CCL5-AS remains to be elucidated in a future study.

In conclusion, the expression levels of CCL5-AS were lower than that of CCL5-reference in normal and mastitis mammary tissues, and a novel transcript of CCL5-AS was detected with a deletion of 112 bp compared with CCL5-reference. Two novel SNPs, SNP g.1647C $>$ T and SNP g.1804G $>$ A were identified, but the CCL5 alternative splicing was not associated with SNP g.1647C $>$ T and SNP g.1804G >A.

\section{Acknowledgements}

Not applicable.

\section{Funding}

This work was supported by grants from the National Natural Science Foundation of China (grant no. 31672397), Shandong Provincial Natural Science Foundation for Distinguished Young Scholars of China (grant no. JQ201709), Major Project of National Transgene in China (grant no. 2018ZX08007001-002), Shandong Provincial Natural Science Foundation (grant no. ZR2016CM37), Shandong Science and Technological Development Plan (grant no. 2013GNC11023), and China Agriculture Research System (grant no. CARS-36).

\section{Availability of data and materials}

The datasets used and/or analyzed during the current study are available from the corresponding author on reasonable request.

\section{Authors' contributions}

JH, LY and RG conceptualized the study design. RG, ZJ, $\mathrm{XW}$ and QJ performed the experiments. YL, HZ, KH and JL analyzed the data. LY and JH wrote the paper.

\section{Ethics approval and consent to participate}

This study was approved by the Animal Care and Use Committee from the Dairy Cattle Research Center, Shandong Academy of Agricultural Sciences (Jinan, China).

\section{Patient consent for publication}

Not applicable.

\section{Competing interests}

The authors declare that they have no competing interests.

\section{References}

1. Eshetu E: An overview on the epidemiology and diagnosis of bovine mastitis. Adv Life Sci Technol 35: 23-27, 2015.

2. Olde Riekerink RG, Barkema HW, Kelton DF and Scholl DT: Incidence rate of clinical mastitis on Canadian dairy farms. J Dairy Sci 91: 1366-1377, 2008.

3. Wang X, Zhong J, Gao Y, Ju Z and Huang J: A SNP in intron 8 of CD46 causes a novel transcript associated with mastitis in Holsteins. BMC Genomics 15: 630, 2014.

4. Bannerman DD, Paape MJ, Lee JW, Zhao X, Hope JC and Rainard P: Escherichia coli and Staphylococcus aureus elicit differential innate immune responses following intramammary infection. Clin Diagn Lab Immunol 11: 463-472, 2004.

5. Swanson KM, Stelwagen K, Dobson J, Henderson HV, Davis SR, Farr VC and Singh K: Transcriptome profiling of Streptococcus uberis-induced mastitis reveals fundamental differences between immune gene expression in the mammary gland and in a primary cell culture model. J Dairy Sci 92: $117-129,2009$.

6. Wang XG, Ju ZH, Hou MH, Jiang Q, Yang CH, Zhang Y, Sun Y, Li RL, Wang CF, Zhong JF and Huang JM: Deciphering transcriptome and complex alternative splicing transcripts in mammary gland tissues from cows naturally infected with Staphylococcus aureus mastitis. PLoS One 11: e0159719, 2016.

7. Lahouassa H, Moussay E, Rainard P and Riollet C: Differential cytokine and chemokine responses of bovine mammary epithelial cells to Staphylococcus aureus and Escherichia coli. Cytokine 38: 12-21, 2007.

8. Gilbert FB, Cunha P, Jensen K, Glass EJ, Foucras G, Robert-Granié C, Rupp R and Rainard P: Differential response of bovine mammary epithelial cells to Staphylococcus aureus or Escherichia coli agonists of the innate immune system. Vet Res 44: 40, 2013.

9. Aldinucci D and Colombatti A: The inflammatory chemokine CCL5 and cancer progression. Mediators Inflamm 2014: 292376, 2014.

10. Soria G and Ben-Baruch A: The inflammatory chemokines CCL2 and CCL5 in breast cancer. Cancer Lett 267: 271-285, 2008.

11. Wu J, Akerman M, Sun S, McCombie WR, Krainer AR and Zhang MQ: SpliceTrap: A method to quantify alternative splicing under single cellular conditions. Bioinformatics 27: 3010-3016, 2011.

12. Baralle FE and Giudice J: Alternative splicing as a regulator of development and tissue identity. Nat Rev Mol Cell Biol 18: 437-451, 2017.

13. Chacko E and Ranganathan S: Genome-wide analysis of alternative splicing in cow: Implications in bovine as a model for human diseases. BMC Genomics 10 (Suppl 3): S11, 2009.

14. Montgomery SB, Sammeth M, Gutierrez-Arcelus M, Lach RP, Ingle C, Nisbett J, Guigo R and Dermitzakis ET: Transcriptome genetics using second generation sequencing in a Caucasian population. Nature 464: 773-777, 2010.

15. Garcia-Blanco MA, Baraniak AP and Lasda EL: Alternative splicing in disease and therapy. Nat Biotechnol 22: 535-546, 2004.

16. Ju Z, Wang C, Wang X, Yang C, Sun Y, Jiang Q, Wang F, Li M, Zhong $\mathrm{J}$ and Huang J: Role of an SNP in alternative splicing of bovine NCF4 and mastitis susceptibility. PLoS One 10: e0143705, 2015.

17. Zhang Z, Wang X, Li R, Ju Z, Qi C, Zhang Y, Guo F, Luo G, Li Q, Wang C, et al: Genetic mutations potentially cause two novel NCF1 splice variants up-regulated in the mammary gland, blood and neutrophil of cows infected by Escherichia coli. Microbiol Res 174: 24-32, 2015.

18. Ju Z, Wang C, Li Q, Hou M, Gao S, Hou Q, Li J, Huang J and Zhong J: Alternative splicing and mRNA expression analysis of bovine SLAMF7 gene in healthy and mastitis mammary tissues. Mol Biol Rep 39: 4155-4161, 2012.

19. Livak KJ and Schmittgen TD: Analysis of relative gene expression data using real-time quantitative PCR and the 2(-Delta Delta C(T)) method. Methods 25: 402-408, 2001. 
20. Mount JA, Karrow NA, Caswell JL, Boermans HJ and Leslie KE: Assessment of bovine mammary chemokine gene expression in response to lipopolysaccharide, lipotechoic acid + peptidoglycan, and CpG oligodeoxynucleotide 2135. Can J Vet Res 73:49-57, 2009.

21. Sipka A, Klaessig S, Duhamel GE, Swinkels J, Rainard P and Schukken Y: Impact of intramammary treatment on gene expression profiles in bovine Escherichia coli Mastitis. PLoS One 9 e85579, 2014

22. Pakianathan DR, Kuta EG, Artis DR, Skelton NJ and Hébert CA: Distinct but overlapping epitopes for the interaction of a CC-chemokine with CCR1, CCR3 and CCR5. Biochemistry 36: 9642-9648, 1997.

23. Marques RE, Guabiraba R, Russo RC and Teixeira MM: Targeting CCL5 in inflammation. Expert Opin Ther Targets 17: 1439-1460, 2013

24. Keren H, Lev-Maor G and Ast G: Alternative splicing and evolution: Diversification, exon definition and function. Nat Rev Genet 11: 345-555, 2010.

25. Swami M: Alternative splicing: Deciding between the alternatives. Nat Rev Genet 10:71, 2009.

26. Wang ET, Sandberg R, Luo S, Khrebtukova I, Zhang L, Mayr C, Kingsmore SF, Schroth GP and Burge CB: Alternative isoform regulation in human tissue transcriptomes. Nature 456: 470-476, 2008 .
27. Manning KS and Cooper TA: The roles of RNA processing in translating genotype to phenotype. Nat Rev Mol Cell Biol 18: 102-114, 2017.

28. Hsiao YH, Bahn JH, Lin X, Chan TM, Wang R and Xiao X: Alternative splicing modulated by genetic variants demonstrates accelerated evolution regulated by highly conserved proteins. Genome Res 26: 440-450, 2016.

29. Latorre E and Harries LW: Splicing regulatory factors, ageing and age-related disease. Ageing Res Rev 36: 165-170, 2017.

30. Stankiewicz P and Lupski JR: Structural variation in the human genome and its role in disease. Annu Rev Med 61: 437-455, 2010.

31. Yang H, Zhong Y, Peng C, Chen JQ and Tian D: Important role of indels in somatic mutations of human cancer genes. BMC Med Genet 11: 128, 2010

32. Huang XF, Huang ZQ, Fang XL, Chen ZJ, Cheng W and Jin ZB: Retinal miRNAs variations in a large cohort of inherited retinal disease. Ophthalmic Genet 39: 175-179, 2018.

33. Huang XF, Mao JY, Huang ZQ, Rao FQ, Cheng FF, Li FF, Wang QF and Jin ZB: Genome-wide detection of copy number variations in unsolved inherited retinal disease. Invest Ophthalmol Vis Sci 58: 424-429, 2017. International (CC BY-NC-ND 4.0) License. 